\title{
Tratamento cirúrgico de lesão do ligamento cruzado anterior em paciente com imaturidade esquelética pela técnica de Kocher, com seguimento pós operatório de 6 anos: relato de caso
}

\author{
Surgical treatment of anterior cruciate ligament injury in a patient with skeletal immaturity \\ by the Kocher technique, with a postoperative follow-up of 6 years: Case report
}

Alfredo dos Santos Netto ${ }^{\circledR}$, Leticia Sano Kawano ${ }^{\circledR 2}$, Victor Marques de Oliveira ${ }^{\circledR}$, Nilson Roberto Severino ${ }^{\circledR 1}$, Osmar Pedro Arbix de Camargo ${ }^{\circledR 1}$, Ricardo de Paula Leite Cury ${ }^{\circledR 1}$

\section{Resumo}

Introdução: No tratamento cirúrgico da lesão do ligamento cruzado anterior (LCA) em pacientes pediá-tricos, é necessário o equilíbrio entre a restauração da anatomia, e o risco de dano à fise de crescimento durante o ato operatório. Objetivo: Relatar caso de paciente com 9 anos, de-senvolvimento puberal de Tanner I, submetido a reconstrução do LCA pela técnica de Ko-cher, técnica de reconstrução extra fisária com enxerto de trato iliotibial autólogo.

Palavras chave: Joelho, Reconstrução do ligamento cruzado anterior, Maturidade sexual

\begin{abstract}
Introduction: In the surgical treatment of anterior cruciate ligament (ACL) injury in pediatric patients, a balance between anatomical restoration and the risk of damage to the growth plate during the surgery is necessary. Objective: Report a case of a 9-year-old patient, pubertal development of Tanner I, underwent ACL reconstruction using the technique of Kocher et al., a physeal-sparing reconstruction technique with autologous iliotibial tract graft.
\end{abstract}

1. Faculdade de Ciência Médicas da Santa Casa de São Paulo. Departamento de Ortopedia e Traumatologia. São Paulo-SP - Brasil 2. Irmandade da Santa Casa de Misericórdia de São Paulo. Departamento de Ortopedia. São Paulo-SP - Brasil

Trabalho realizado: Faculdade de Ciência Médicas da Santa Casa de São Paulo. Departamento de Ortopedia e Traumatologia. São Paulo-SP - Brasil

Endereço para correspondência: Alfredo dos Santos Netto. Rua Pamplona, 145 cj 807 - Jardim Paulista-01405-900 - São PauloSP - Brasil.E-mail: alfredo.santos@fcmsantacasasp.edu.br Os autores declaram que não tem nenhum conflito de interesse.
Keywords: Knee, Anterior cruciate ligament reconstruction, Sexual maturation

\section{Introdução}

No tratamento cirúrgico da lesão do ligamento cruzado anterior (LCA) em pacientes pediátricos, é necessário o equilíbrio entre a restauração da anatomia e o risco de dano à fise de crescimento durante a cirurgia, o que pode resultar em discrepância de comprimento e/ou deformidades angulares ${ }^{(1)}$.

Várias técnicas cirúrgicas foram descritas para reconstrução do LCA nessa população. Kocher et $\mathrm{al}^{(2)}$ descreveram uma reconstrução do LCA, com uso de enxerto autólogo do trato iliotibial, poupando a fise de crescimento, com bons resultados.

Reportamos o caso de um paciente que sofreu lesão do LCA com 9 anos de idade e foi submetido à reconstrução pela técnica de Kocher, com seguimento pós operatório de 6 anos.

\section{Relato de Caso}

O relato foi devidamente submetido ao Comitê de Ética com aprovação - CAAE: 34741720.8.0000.5479 - número de aprovação 4.217.140 - Santa Casa de Misericórdia de São Paulo.

\section{Apresentação pré operatória}

Paciente com 9 anos e 6 meses na época da lesão, do sexo masculino, Tanner I, sofreu trauma torcional no joelho esquerdo durante prática esportiva (futebol). Evoluiu com dor e instabilidade, que limitava as atividades diárias. No exame físico apresentava instabilidade anterior do joelho (teste de Lachman $3+/ 3+$, teste do pivot shift $2+/ 3+$, teste da gaveta an- 
Santos Netto A, Kawano LS, Oliveira VM, Severino NR, Camargo OPA, Cury RPL. Tratamento cirúrgico de lesão do ligamento cruzado anterior em paciente com imaturidade esquelética pela técnica de Kocher, com seguimento pós operatório de 6 anos: relato de caso. Arq Med Hosp Fac Cienc Med Santa Casa São Paulo. 2021; 66:e022.

terior $2+/ 3+$, rolímetro com diferença maior que $3 \mathrm{~mm}$ comparado com lado contralateral, e testes meniscais para lesão do menisco medial positivos. Na avaliação funcional, o Lysholm pré operatório foi de 53 (ruim), IKDC objetivo como D (muito anormal), e Tegner pré operatório de 3 (trabalho leve).

Paciente realizou ressonância magnética que evidenciou lesão no LCA e lesão longitudinal no corno posterior do menisco medial (figura 1).
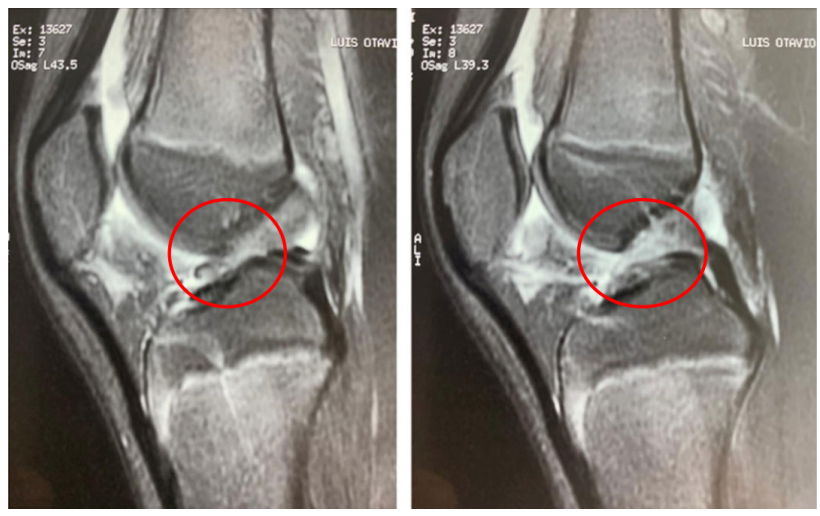

Figura 1 - Imagem de ressonância magnética pré-operatória em corte sagital, evidenciando lesão completa no ligamento cruzado anterior.

Dois meses após a lesão, foi submetido a reconstrução do LCA pela técnica de Kocher et al² além de sutura do menisco medial (técnica de dentro para fora).

\section{Técnica Cirúrgica}

Na técnica de Kocher et al ${ }^{(2)}$ é realizada uma incisão de aproximadamente $6 \mathrm{~cm}$ de comprimento a partir da linha articular lateral do joelho. Para retirada do enxerto do trato iliotibial, realiza-se incisões ao longo das suas margens superior e inferior a partir do tubérculo de Gerdy até 15 a $20 \mathrm{~cm}$ proximais à linha articular. A banda iliotibial é destacada proximalmente e dissecada da cápsula lateral, e suturada em chuleio com Ethibond $\mathrm{n}^{\circ} 5$. O enxerto do trato iliotibial é direcionado para dentro do joelho, passado pelo topo do côndilo femoral lateral, em posição "over the top", e em seguida é levado em direção à tíbia, passando sob o ligamento intermeniscal. O enxerto é fixado no lado femoral através da incisão lateral com o joelho em $90^{\circ}$ de flexão, e no lado tibial é realizado a sutura no periósteo sob tensão.

\section{Apresentação pós operatória}

Após protocolo de reabilitação de seis meses pós-operatório, paciente teve boa evolução, retornando para suas práticas esportivas.

Com cinco anos de pós operatório, apresentou queixa de dor após atividade esportiva, sendo diagnosticado nova lesão do menisco medial. Foi submetido a artroscopia para meniscectomia parcial, onde foi evidenciado aspecto normal do enxerto do ligamento cruzado anterior (figura 2).

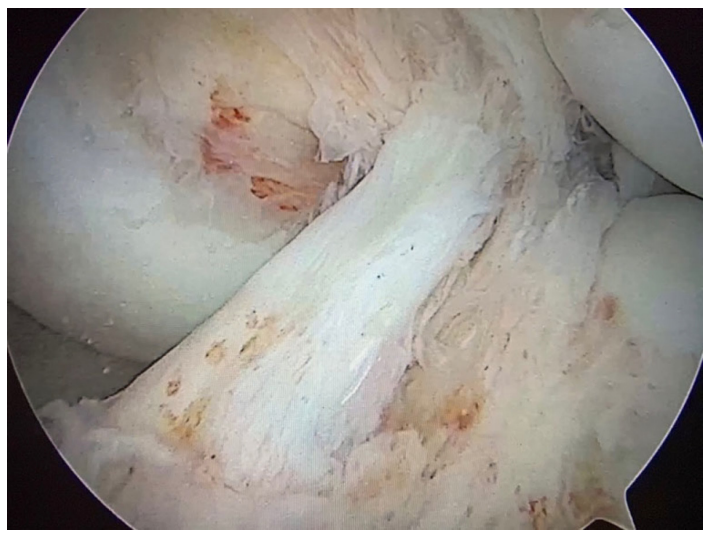

Figura 2 - Imagem intra-operatória da artroscopia para tratamento de lesão meniscal, realizada cinco anos após a cirurgia inicial de reconstrução ligamentar, evidenciado integridade do enxerto do ligamento cruzado anterior.

Segue em acompanhamento, com seguimento pós-operatório de 6 anos da primeira cirurgia. Nega queixas, porém observamos discreto valgo assimétrico a esquerda. Apresenta teste de Lachman negativo, teste do pivot shift negativo, teste da gaveta anterior negativo, rolímetro com diferença de $1 \mathrm{~mm}$ do lado contralateral, sem alteração do arco de movimento (flexão 130 graus e extensão 0 graus bilateral).

Foram aplicados questionários para avaliação funcional do joelho que demostraram Lysholm de 100 (excelente), IKDC objetivo como A (normal), e Tegner de 9 (esportes competitivos).

$\mathrm{Na}$ avaliação dos exames de imagens atuais, observarmos enxerto do LCA íntegro na ressonância magnética (figura 3), e desvio de $4,37^{\circ}$ (Bonessetter Apps
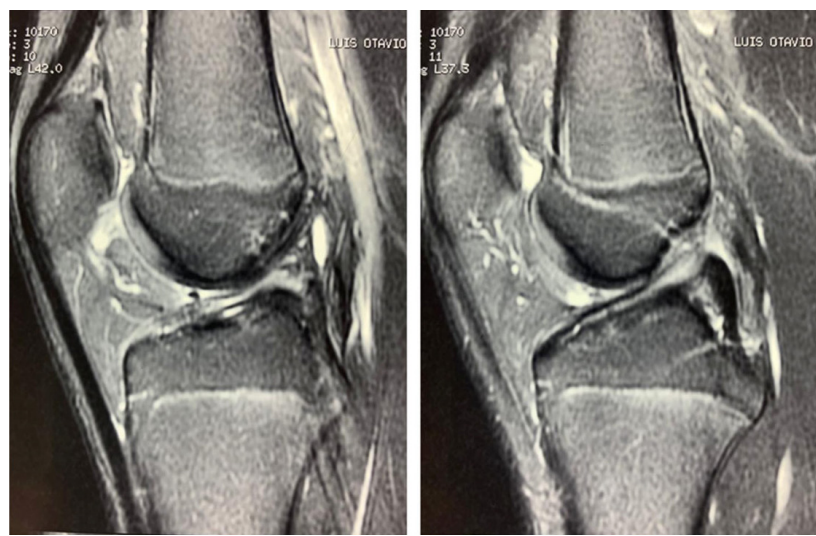

Figura 3 - Imagem de ressonância magnética em corte sagital, realizada com 6 anos de pós-operatório, evidenciando integridade do enxerto da reconstrução do ligamento cruzado anterior. 
LLc built) em valgo no lado esquerdo, assimétrico em relação ao lado contralateral, nas radiografias panorâmicas com carga dos membros inferiores (figura 4).

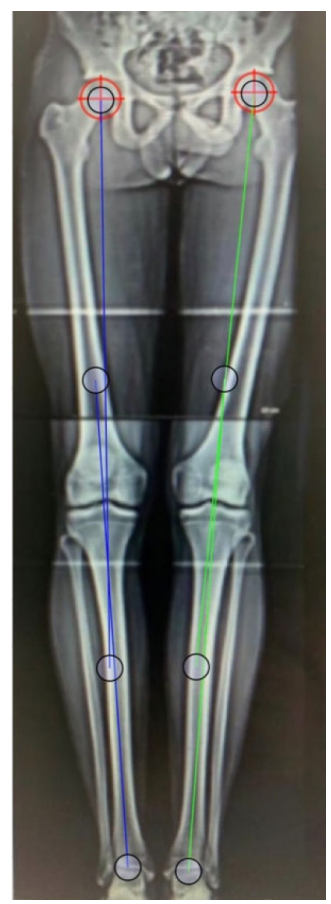

Figura 4 - Imagem de radiografia panorâmica dos membros inferiores evidenciando eixo mecânico a direita de $177,13^{\circ}$ e a esquerda de $181,5^{\circ}$, desvio de $4,37^{\circ}$ em valgo no lado esquerdo. Utilizado aplicativo Bonessetter Apps LLc built

\section{Discussão}

Historicamente, o tratamento não cirúrgico ou aguardar até o término da maturidade esquelética para realizar a reconstrução do LCA pelas técnicas convencionais eram as estratégias de tratamento mais utilizadas nessa lesão. Atualmente, o entendimento do risco de lesões meniscais e condrais resultantes da instabilidade, motiva a indicação de tratamento cirúrgico em caso de lesão do LCA, mesmo em pacientes com esqueleto imaturo, nos quais existe maior risco de ruptura do enxerto, além de dano à fise de crescimento no ato operatório ${ }^{(3-6)}$.

Não existe um consenso na literatura, mas o algoritmo mais utilizado indica que pacientes pré-puberais (estagio 1 ou 2 de Tanner) devam ser submetidos a reconstrução extra fisária ou epifisárias enquanto pacientes adolescentes (estagio 3 ou superior de Tanner) estariam indicadas técnicas transfisárias parciais ou totais, com utilização de enxerto de tecidos moles, com fixação metafisária( ${ }^{(7)}$.

A reruptura, as deformidades angulares e a disprepância de membros inferiores figuram como as complicações mais comuns. Wong et al ${ }^{(8)}$, em metanálise reviram 1372 pacientes submetidos a reconstrução com técnicas transfisárias ou não, com média de 13 anos de seguimento, encontraram $8,7 \%$ de reruptura, $3,7 \%$ desenvolveram deformidade angulares, mais comum em valgo, e 7,5\% de discrepância dos membros inferiores, sendo as duas últimas complicações mais frequentes em técnicas transfisárias. Já a técnica proposta por Kocher et $\mathrm{al}^{(9)}$ mostrasse efetiva ao tentar evitar tais complicações. Com seguimento médio de 5,3 anos, em 237 pacientes, os autores não encontraram deformidade angular ou discrepância dos membros em nenhum paciente avaliado, e índice de reruptura de 6,6 \%, com IKDC de 93, Lysholm de 93,4 e tegner de 7,8 na avaliação final. Apesar do não posicionamento anatômico dos túneis, a reconstrução combinada intra e extraarticular, semelhante a reconstrução do ligamento anterolateral, ajudaria a explicar os bons resultados quanto a estabilidade e baixo índice de reruptura, segundo os autores.

Nosso paciente apesar do leve desvio em valgo encontrado na avaliação final, apresentou escores funcionais semelhantes aos resultados de Kocher et $\mathrm{al}^{(9)}$, com Lysholm de 100 (excelente), IKDC objetivo como A (normal), e Tegner de 9, comprovando a efetividade da técnica.

\section{Conclusão}

Esse caso enfatiza a possibilidade de bom resultado funcional em paciente Tanner 1 submetido a reconstrução do ligamento cruzado anterior pela técnica de Kocher, mas também alerta o risco de deformidade angular no pós operatório, mesmo utilizando técnica poupadora da fise.

\section{Referências}

1. Ardern CL, Ekås G, Grindem H, Moksnes H, Anderson A, Chotel FB, et al. 2018 International Olympic Committee consensus statement on prevention, diagnosis and management of paediatric anterior cruciate ligament (ACL) injuries. Knee Surg Sports Traumatol Arthrosc. 2018; 26(4):989-1010.

2. Kocher MS, Garg S, Micheli LJ. Physeal sparing reconstruction of the anterior cruciate ligament in skeletally immature prepubescent children and adolescents. Surgical technique. J Bone Joint Surg Am. 2006; 88(Suppl 1 Pt 2):283-93.

3. Astur DC, Cachoeira CM, Vieira TS, Debieux P, Kaleka CC, Cohen M. Increased incidence of anterior cruciate ligament revision surgery in pediatric verses adult population. Knee Surg Sports Traumatol Arthrosc. 2018; 26(5):1362-6.

4. Astur DC, Novaretti JV, Cavalcante ELB, Goes A Jr, Kaleka CC, Debieux $\mathrm{P}$, et al. Pediatric anterior cruciate ligament reruptures are related to lower functional scores at the time of return to activity: a prospective, midterm follow-up study. Orthop J Sports Med. 2019; 7(12):2325967119888888.

5. Wall EJ, Ghattas PJ, Eismann EA, Myer GD, Carr P. Outcomes and complications after all-epiphyseal anterior cruciate ligament reconstruction in skeletally immature patients. Orthop J Sports Med. 2017; 5(3):2325967117693604. 
6. Demange MK, Camanho GL. Nonanatomic anterior cruciate ligament reconstruction with double-stranded semitendinosus grafts in children with open physes: minimum 15-year followup. Am J Sports Med. 2014; 42(12):2926-32.

7. Fabricant PD, Kocher MS. Management of ACL injuries in children and adolescents. J Bone Joint Surg Am. 2017;99(7):60012.

8. Wong SE, Feeley BT, Pandya NK. Complications after pediatric ACL reconstruction: a meta-analysis. J Pediatr Orthop. 2019;39(8):e566-71.
9. Kocher MS, Heyworth BE, Fabricant PD, Tepolt FA, Micheli LJ. Outcomes of physeal-sparing acl reconstruction with iliotibial band autograft in skeletally immature prepubescent children. J Bone Joint Surg Am. 2018; 100(13):1087-94.

Trabalho recebido: $24 / 05 / 2021$

Trabalho aprovado: $12 / 06 / 2021$

Trabalho publicado: 02/08/2021 